\title{
Implementation of ARIMA model to predict Rain Attenuation for KU-band 12 Ghz Frequency
}

\author{
Dhaval P. Patel ${ }^{1}$, Mitul M. Patel ${ }^{2,}$ Devendra R. Patel ${ }^{3}$ \\ ${ }^{I}$ (Electronics and communication Department, Parul Institute of Engg. \& Tech.,India) \\ ${ }^{2}$ (Electronics and communication Department, Parul Institute of Engg. \& Tech.,India) \\ ${ }^{3}$ (Electronics and communication Department, Parul Institute of Tech., India)
}

\begin{abstract}
Satellite communication systems operating at Ka band and Ku band frequencies must affect the problem of propagation impairments which includes fading due to rain, clouds, snow for obtaining the required performance. Rain affects the signal most and involved is absorption \& scattering. Auto-Regressive Integrated Moving Average (ARIMA) model is used to generate and predict the time series values for rain attenuation. The predicted values obtained for different window size of the ARIMA model and analysis is done in terms of error matrix
\end{abstract}

Keywords: ARMA model, ARIMA model, KU-band, Time series, Time series analysis.

\section{INTRODUCTION}

A time series is a collection of data points which are generally sampled equally in time intervals. Time series prediction refers to the process by which the values of a system is forecasted based on the information obtained from the past and current data points.In statistics, signal processing, econometrics and mathematical finance, a time series is a sequence of data points, measured typically at successive time instants spaced at uniform time intervals. Examples of time series are the daily closing value of the Dow Jones index or the annual flow volume of the Nile River at Aswan.

Time series analysis comprises methods for analyzing time series data in order to extract meaningful statistics and other characteristics of the data. Time series forecasting is the use of a model to predict future values based on previously observed values. Time series are very frequently plotted via line charts.

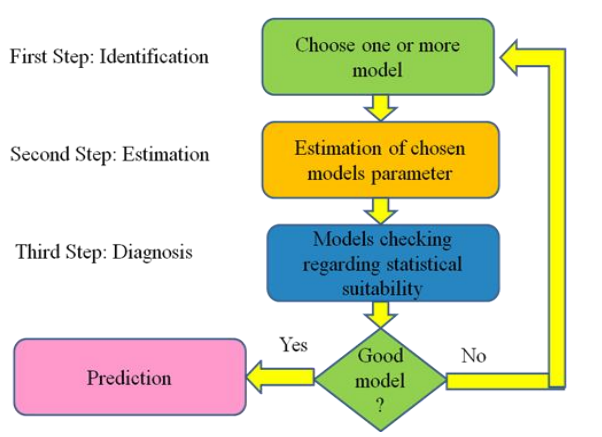

Fig.1 Block diagram of model selection procedure

In general, time series predictability is a measure of how well future values of a time series can be forecasted, where a time series is a sequence of observations $\{\mathrm{Yt}, \mathrm{t}=1,2, \ldots \ldots, \mathrm{N}\}$. Time series predictability indicates to what extent the past can be used to determine the future in a time series. A time series generated by a deterministic linear process has high predictability, and its future values can be forecasted very well from the past values. [13]

RADIO waves above $10 \mathrm{GHz}$ are highly influenced by rain induced attenuation, not just the magnitude thereof, but also the rate of its variation. In order to achieve a reliable design of wireless communication systems operating at these frequencies, it is necessary to evaluate the design in a simulation against time series of rain attenuation generated from a realistic model.[1], [2].Rain attenuation is one of the important impairments that affects RF signal at $\mathrm{Ku}$ and $\mathrm{Ka}$ band frequencies. The forecasting of the rainfall and rain attenuation plays a vital role in the fields of communications, agriculture, military services, etc. Number of models have been proposed and developed for prediction and generation of time series data of rain attenuation based on auto-regressive moving average processes. [6] 
The ARMA model can only be used for stationary time series data and hence cannot be used for nonstationary rain events. Therefore, a step is required to convert the nonstationary data into stationary ones, which is available in auto-regressive integrated moving average (ARIMA) in the form of data differencing [2].

\section{Autoregressive model}

\section{TIME SERIES MODELS}

A time series $\{X t\}$ is said to be an autoregressive process of order $p$ (abbreviated AR $(p)$ ) if it is a weighted linear sum of the past $p$ values plus a random shock so that

$$
X_{t}=\varphi_{1} X_{t-1}+\varphi_{2} X_{t-2}+\cdots+\varphi_{p} X_{t-p}+Z_{t}
$$

Equation. (1)

Where $\left\{Z_{t}\right\}$ denotes a purely random process with zero mean and variance $\sigma_{Z}^{2}$

2. Moving average (MA) model

A time series $\{X t\}$ is said to be a moving average process of order $q$ (abbreviated MA $(q)$ ) if it is a weighted linear sum of the last $q$ random shocks so that

$$
X_{t}=Z_{t}+\theta_{1} Z_{t-1}+\cdots+\theta_{q} Z_{t-q}
$$

Equation. (2)

Where $\left\{Z_{t}\right\}$ denotes a purely random process with zero mean and constant variance $\sigma_{z}^{2}$.

\section{Auto regressive moving average (ARMA) model}

A mixed autoregressive moving average model with $\mathrm{p}$ autoregressive terms and $\mathrm{q}$ moving average terms is abbreviated ARMA (p, q) and may be written as

$$
\varphi(\mathrm{B}) \mathrm{Xt}=\theta(\mathrm{B}) \mathrm{Zt}
$$

Where $\varphi(B), \theta(B)$ are polynomials in B of finite order $p, q$, respectively.[14]

The importance of ARMA processes is that many real data sets may be approximated in a more parsimonious way (meaning fewer parameters are needed) by a mixed ARMA model rather than by a pure AR or pure MA process. Autoregressive-moving-average (ARMA) models are mathematical models of the persistence, or autocorrelation, in a time series. ARMA models are widely used in hydrology, dendrochronology, econometrics, and other fields. There are several possible reasons for fitting ARMA models to data. [14]

ARMA models are widely used for prediction of economic and industrial time series. ARMA models can also be used to remove persistence. In dendrochronology, for example, ARMA modeling is applied routinely to generate residual chronologies - time series of ring-width index with no dependence on past values. This operation, called prewhitening, is meant to remove biologically-related persistence from the series so that the residual may be more suitable for studying the influence of climate and other outside environmental factors on tree growth.[14]

Autoregressive-moving-average (ARMA) models are mathematical models of the persistence, or autocorrelation, in a time series. ARMA models are widely used in hydrology, dendrochronology, econometrics, and other fields. There are several possible reasons for fitting ARMA models to data. ARMA models can be described by a series of equations. The equations are somewhat simpler if the time series is first reduced to zeromean by subtracting the sample mean. Therefore, we will work with the mean-adjusted series

$$
\mathrm{y}_{\mathrm{t}=} \mathrm{Y}_{\mathrm{t}-\mathrm{Y}}, \mathrm{t}=1,2, \ldots \ldots, \mathrm{N} \quad \text { Equation. (4) }
$$

Where $\mathrm{Y}_{\mathrm{t}}$ is the original time series, $\bar{Y}$ is its sample mean, and $\mathrm{y}_{\mathrm{t}}$ is the mean-adjusted series.[14]

\section{ARIMA model}

In practice many (most?) time series are nonstationary and so we cannot apply stationary AR, MA or ARMA processes directly. One possible way of handling non-stationary series is to apply differencing so as to make them stationary. The first differences, namely $(\mathrm{Xt}-\mathrm{Xt}-1)=(1-\mathrm{B}) \mathrm{Xt}$, may themselves be differenced to give second differences, and so on. The dth differences may be written as (1-B) dXt. If the original data series is differenced d times before fitting an $\operatorname{ARMA}(p, q)$ process, then the model for the original indifferences series is said to be an $\operatorname{ARIMA}(p, d, q)$ process where the letter ' $I$ ' in the acronym stands for integrated and d denotes the number of differences taken.

The Box-Jenkins approach to modeling and forecasting time series data is but one of a large family of quantitative forecasting methods which have been developed in the fields of operations research, statistics, and management science. Box-Jenkins models are also known as "ARIMA" models, the acronym standing for Autoregressive Integrated Moving Average. An ARIMA (p, d, q) model of the nonstationary random process $\mathrm{Y}(\mathrm{t})$ is expressed as

$$
\Phi(\mathrm{B})(1-\mathrm{B}) \wedge \mathrm{d} \mathrm{Xt}=\theta(\mathrm{B}) \mathrm{Zt}
$$

with an AR operator

$\Phi_{p}(B)=1-\phi_{1} B-\ldots-\phi_{p} B^{p}$

and a MA operator

$\Theta_{\mathrm{q}}(\mathrm{B})=1-\theta_{1} \mathrm{~B}-\ldots-\theta_{\mathrm{q}} \mathrm{B}^{\mathrm{q}}$

Where $\phi_{p \text { is } p}^{\text {th }}$ AR coefficient, $\theta_{\mathrm{q} \text { the }}{ }^{\text {th }} \mathrm{MA}$ coefficient, $\mathrm{Zt}$ noise, Xt Rain attenuation. [2] 


\section{Simulation Result of MATLAB}

\section{RESULT ANALYSIS}

Here, collected real rain fall data for KU-band satellite from National Weather Service Climate Prediction Centre, Maryland, U.S. Rain Attenuation reading are changing according to Longitude, which is varies from 0 to 360 and Latitude is fix -11.25 and 144 samples are taken from January, 2012. Here frequency is $12 \mathrm{GHz}$. Thus Plot is Longitude vs. Rain Attenuation. ARIMA model is used to predict and generate the time series of rain attenuation for a given set of parameters.

Figure 2,3,4,5 are show the plot of rain attenuation with different window size 1,2,5,10 respectively.

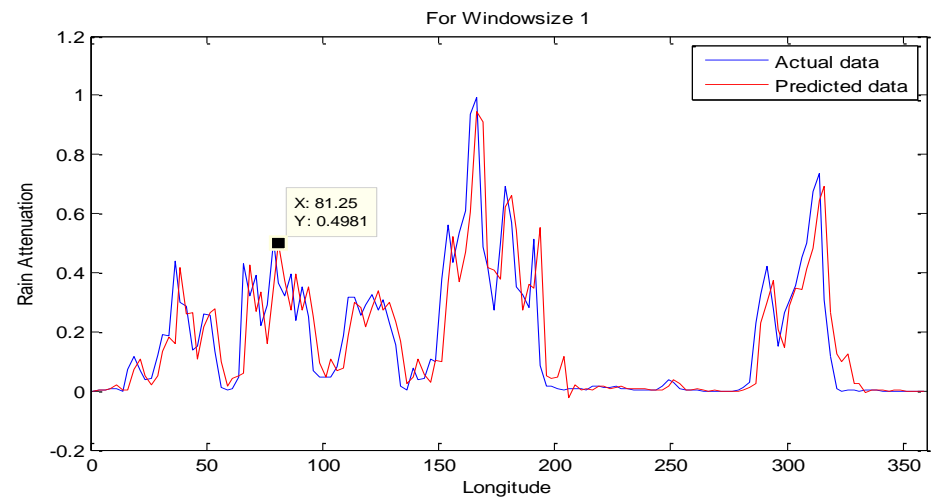

Fig. 2 Plot of actual data and Predicted data with window size is 1.

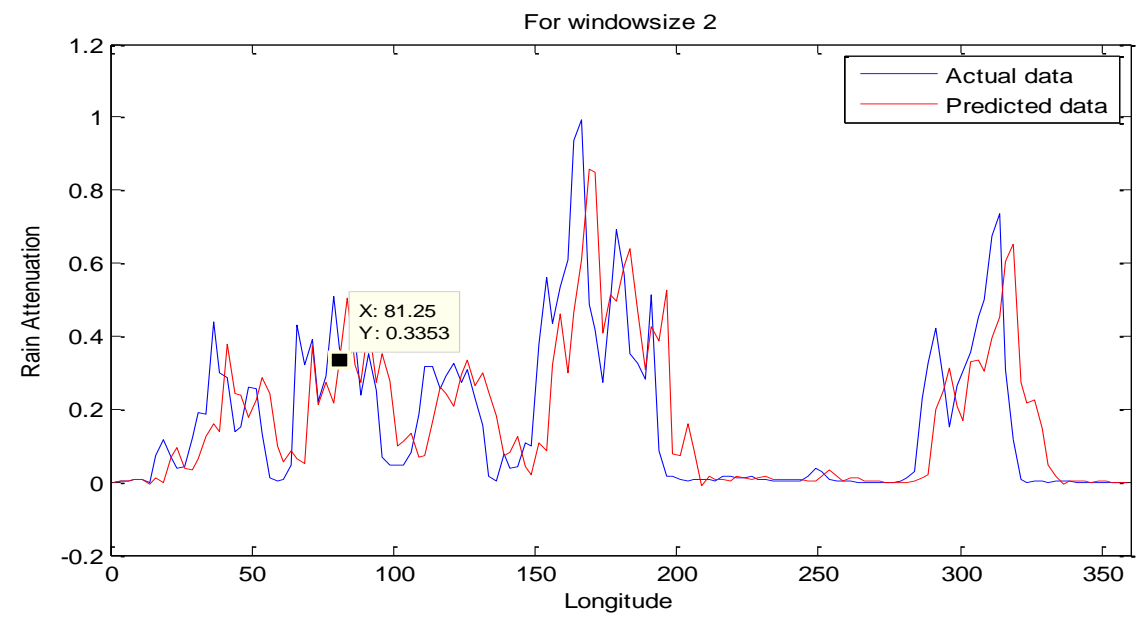

Fig.3 Plot of actual data and Predicted data with window size is 2.

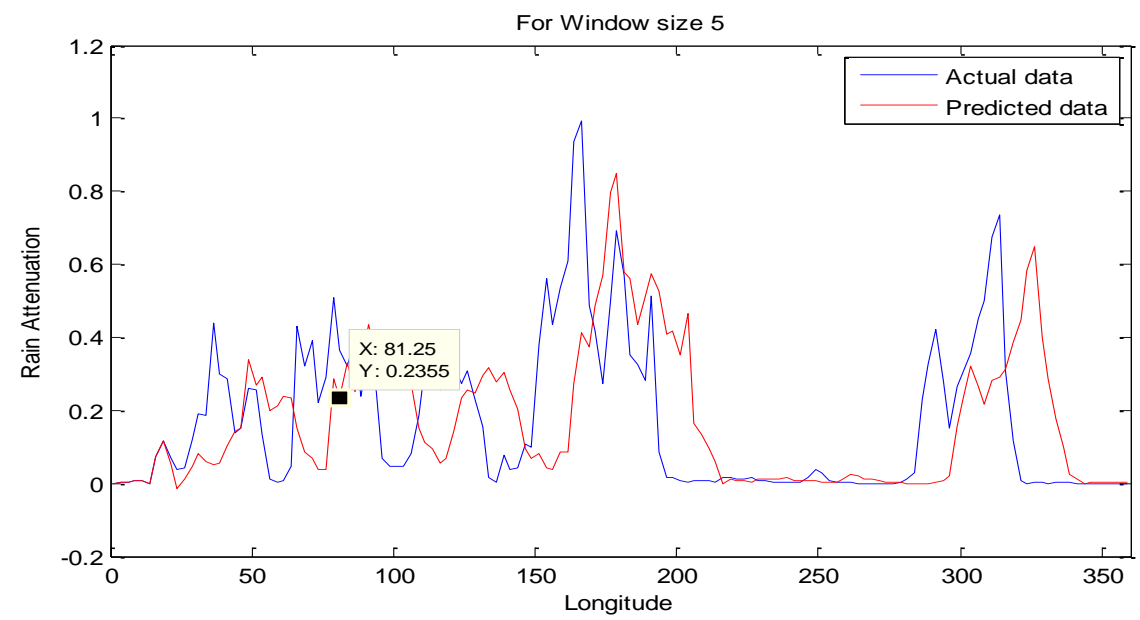

Fig.4 Plot of actual data and Predicted data with window size is 5. 


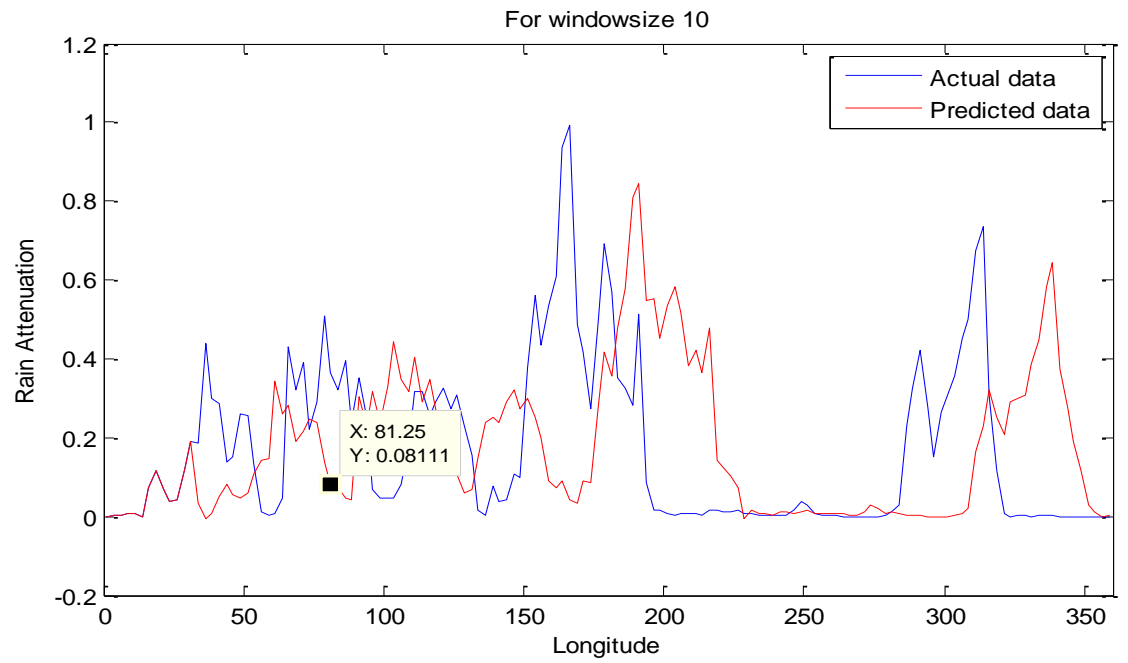

Fig.5 Plot of actual data and Predicted data with window size is 10.

Table 1: Reading of Error Matrix

\begin{tabular}{|c|c|c|c|c|}
\hline \multirow{2}{*}{ Error } & \multicolumn{4}{|c|}{ ARIMA model Predicted output } \\
\cline { 2 - 5 } & Window size 1 & Window size 2 & Window size 5 & Window size 10 \\
\hline MSE & 0.000085 & 0.000178 & 0.000339 & 0.000525 \\
\hline RMSE & 0.009200 & 0.013300 & 0.018400 & 0.022900 \\
\hline MAPE & 0.000001 & 0.000001 & 0.000002 & 0.000004 \\
\hline
\end{tabular}

The table 1 shows the error of Rain Attenuation which is predicted by ARIMA model. We have observed that ARIMA model is best for this application because error is very less but, error is increase with respect to increasing window size.

\section{CONCLUSION}

We have collected real rain fall data for KU-band satellite from National Weather Service Climate Prediction Centre, Maryland, U.S. we have studied different types of prediction model. In this paper, the results of rain attenuation are generated using by ARIMA model. ARIMA model is best for prediction of rain attenuation for Ku-band satellite for $12 \mathrm{GHz}$ frequency because error is very less. Here we check the error by using error matrix (MSE, RMSE, and MAPE) which is increase with respect to increasing window size.

\section{Papers:}

\section{REFERENCES}

[1] M. Sridhar 1, K. Padma Raju2, Ch. Srinivasa Rao3, D. Venkata Ratnam1,“ Prediction and Analysis of Rain Attenuation using ARIMA Model at Low latitude Tropical Station”,International Journal of Advanced Research in Electrical, Electronics and Instrumentation Engineering Vol. 2, Issue 7, July 2013.

[2] A. Mauludiyanto, G. Hendrantoro, Member, IEEE, M. H. Purnomo, Member, IEEE, T. Ramadhany, and A. Matsushima, Member, IEEE “ARIMA Modeling of Tropical Rain Attenuation on a Short 28-GHz Terrestrial Link" IEEE ANTENNAS AND WIRELESS PROPAGATION LETTERS, VOL. 9, 2010.

[3] R. Billinton, H. Chen and R. Ghajar, "Time-series models for reliability evaluation of power systems including wind energy,"Micro electron. Reliab., vol. 36, pp. 1253-1261, 1996.

[4] Jie YANG, Zenliang GAO, Jianbin TANG, Ying CHEN, "A Practical Predictive Control Algorithm for Embedded System and Its Application", Journal of Computational Information Systems 6:11, 3501-3508, 2010

[5] M. Shafie-khah1, M. Parsa Moghaddam , M.K. Sheikh-El-Eslami2.” Price forecasting of day-ahead electricity markets using a hybrid forecast method." 15 October 2010 Energy Conversion and Management 52 (2011) 2165-2169

[6] G. Hendrantoro, A. Mauludiyanto, and P. Handayani, "An autoregressive model for simulation of time-varying rain rate," in Proc. Symp. Antenna Appl. Electromagn./URSI Conf., Ottawa, ON, Canada, Jul. 20-23, pp. 613-616, 2004.

[7] Yanhua YU, Jun WANG, Xiaosu ZHAN, Junde SONG,“ Novel anomaly detection approach for telecommunication Network proactive performance monitoring”, Front. Electr. Electron. Eng. China 2009.

[8] Shkelzen CAKAJ "Rain Attenuation Impact on Performance of Satellite Ground Stations for Low Earth Orbiting (LEO) Satellites in Europe" Int. J. Communications, Network and System Sciences, 2009.

[9] Bo Wanga, Neng-ling Taia,*, Hai-qing Zhaib, Jian Yeb, Jia-dong Zhuc, Liang-bo Qic, "A new ARMAX model based on evolutionary algorithm and particle swarm optimization for short-term load forecasting" Electric Power Systems Research 78 (2008) 1679-1685.

[10] Peiyuan Chen, Troels Pedersen, Birgitte Bak-Jensen and Zhe Chen, "ARIMA based time series model of stochastic wind power generation". 
Implementation of ARIMA model to predict Rain Attenuation for KU-band 12 Ghz Frequency.

\section{Books:}

[11] A. Leon-Garcia, "Probability, Statistics, and Random Process for Electrical Engineering". 3rd Ed. New Jersey: Pearson Prentice Hall, 2009.

\section{Theses:}

[12] Kofi Agyarko Ababio, "Comparative Study Of Stock Price Forecasting Using Arima And Arimax Models", Kwame Nkrumah University of Science and Technology, Kumasi, June 2012.

[13] Minglei Duan, B.S. "TIME SERIES PREDICTABILITY "Faculty of the Graduate School, Marquette University, Milwaukee, Wisconsin April 5, 2002

\section{Link:}

[14] http://www.ltrr.arizona.edu/ dmeko/notes_5.pdf 